\title{
SISTEMAS DE DOSAGEM PARA COQUILHA TATUR*
}

\author{
Evelyn Dayrell Lopes Júnior ${ }^{1}$ \\ Roberto Dutto ${ }^{2}$ \\ Ivete Peixoto Pinheiro ${ }^{3}$ \\ Sandro Cardoso Santos ${ }^{4}$
}

\section{Resumo}

A capacidade de alimentação de sistemas de fundição é uma das características tecnológicas que está correlacionada a resultados qualitativos negativos, como porosidades e deformações geométricas dos produtos fundidos. As ligas com suas características são parte fundamental do processo de alimentação. A metodologia de caracterização da capacidade de alimentação por meio da coquilha Tatur foi utilizada nesse trabalho, para a qual foram desenvolvidos novos processos de dosagem da liga líquida, com o objetivo de prover maior repetitividade, e consequentemente, maior coerência morfológica das amostras produzidas. Para isso, foram desenvolvidos dois novos métodos de dosagem, chamados "método de dosagem dinâmica" e "método de pré-dosagem". Esses foram testados e analisados, sendo suas amostras avaliadas comparativamente com o padrão morfológico teórico. O objetivo da obtenção de amostras coerentes foi atingido, sendo evidenciadas a grande influência das variáveis presentes na etapa de vazamento sobre os resultados das amostras obtidas.

Palavras-chave:Ligas de alumínio; Massalotes; Sistema de canais; Fundição.

\section{Abstract}

\section{DOSING SYSTEMS FOR TATUR MOLD}

The feeding capacity of foundry systems is one of the technological characteristics correlated to harmful qualitative results, such as porosity and geometric deformation of the castings. The alloys and its characteristics arekey part of the feeding process. The characterization methodology of the feeding capacity by Tatur mold was used in this study. Two new methods of liquid alloy dosing were developed in order to provide greater repeatability and consequently greater morphological consistency of the samples. These methods were called "dynamic dosing method" and "pre-dosing method". These have been tested, analyzed and their samples compared to the theoretical morphology. The goal of obtaining consistent samples was achieved and the influences of the process variables present in the pouring step on the samples were highlighted.

Keywords: Aluminum alloy; Risers; Gate systems; Foundry.

1 Engenheiro Industrial Mecânico - CEFET MG, Engenheiro de produtos e processos, Nemak do Brasil, Betim, Minas Gerais, Brasil. Mestrando em Engenharia de Materiais do Centro Federal de Educação Tecnológica de Minas Gerais, Belo Horizonte, Minas Gerais, Brasil.

2 Engenheiro Metalurgista-Politecnicodi Torino, Supervisor de pesquisa e desenvolvimento para a América do Sul, Nemak do Brasil, Betim, Minas Gerais, Brasil.

3 Engenheira Metalurgista - UFMG, Doutorado, Professora, Departamento de Engenharia de Materiais, Centro Federal de Educação Tecnológica de Minas Gerais, Belo Horizonte, Minas Gerais, Brasil.

4 Engenheiro Mecânico - UFU MG, Doutorado, Professor, Departamento de Engenharia Mecânica, Centro Federal de Educação Tecnológica de Minas Gerais, Belo Horizonte, Minas Gerais, Brasil. 


\section{INTRODUÇÃO}

O presente trabalho apresenta o estudo de metodologias para a caracterização de um dos mais importantes aspectos tecnológicos verificados em processos de fundição, a capacidade de alimentação da liga. Essa se traduz no suprimento de metal líquido que ocorre na interface líquido/ sólido, durante sua mudança de fase. Conforme Campbell [1], o fenômeno da contração durante a solidificação é potencialmente o gerador de maiores problemas devido à formação de constituintes sólidos com maiores densidades, que requerem alimentação do metal líquido para compensação da redução volumétrica. A movimentação do líquido para alimentar esses espaços pode ser impedida pelo próprio constituinte sólido.

O método Tatur foi utilizado para a caracterização da capacidade de alimentação das ligas nesse trabalho. Esse consiste no vazamento do metal líquido em coquilha com geometria particular, a qual proporciona amostras com características relacionadas às descontinuidades oriundas dos fenômenos de contração do material, conforme esquematizado na Figura 1. Devido à contração durante a transformação líquidosólido, a forma cônica da parte superior do molde, em ausência de alimentação proveniente de um massalote, promove a formação de macro e micro porosidades [2].

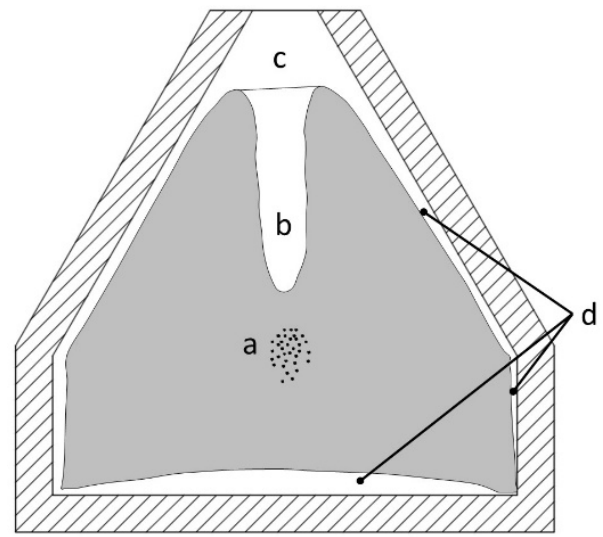

Figura 1. Representação esquemática da coquilha Tatur:

(a) Micro porosidade; (b) Rechupe em vale;(c) Rechupe vertical; (d) Contração geral.

Fonte: La-Orchan [3] - adaptado.

Alguns pesquisadores comentam em seus trabalhos, que a caracterização da capacidade de alimentação das ligas não é usual. Segundo La-Orchan [3], macro e micro porosidades raramente são quantificadas, porém quando são, o teste Tatur é a técnica mais comumente utilizada. Brůna et al. [2] mencionam que para se obter resultados confiáveis é necessário prover um grande número de amostras (cerca de 20) e fazer uma avaliação estatística. A baixa reprodutibilidade das medidas é uma razão adicional para que essa técnica não seja difundida em chão de fábrica.

A disparidade morfológica entre as amostras obtidas e o padrão teórico podem ser observadas na Figura 2, (a) Couper et al. [4], (b) e Brůna et al. [2] e (c) Autor, essasúltimas foram obtidas por vazamentos diretos da concha para o molde, em testes preliminares. 


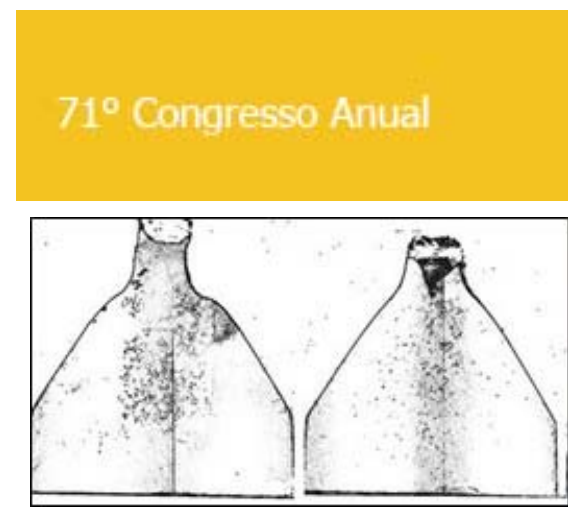

(a)

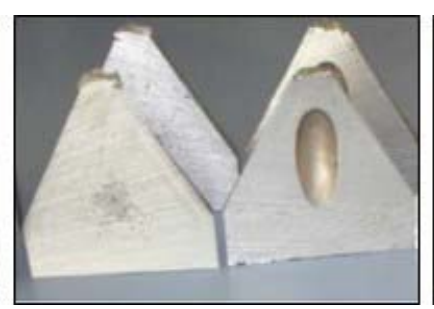

(b)

Figura 2. Exempos de amostras obtidas em alguns trabalhos.

(a) fonte: Couper et.al [4]; (b) fonte: Brůna et al.[2]; (c) fonte: Autor (2013).

As discrepâncias das morfologias observadas entre as amostras produzidas e o padrão teórico do método Tatur foram motivadoras para o desenvolvimento dessa pesquisa. Assim, dois métodos de dosagem do metal para o vazamento foram propostos, com o intuito de reduzir as dispersões do processo, pela influência do operador. Esses foram chamados: "método de dosagem dinâmica" e "método de prédosagem".

\section{MATERIAIS E MÉTODOS}

\subsection{Material da coquilha e liga}

Em seu trabalho, Ahdollahi [5] indica que o material a ser utilizado para a construção da coquilha Tatur deve ser o cobre ou grafite, enquanto Couper [4] utilizou o aço inox. A coquilha Tatur utilizada nesse trabalho foi construída conforme representação esquemática da Figura 3, em aço AISI H13, comumente utilizado para construção de moldes permanentes em fundições de alumínio. Conforme ASM Handbook [6], o aço AISI H13 apresenta boas características tecnológicas, quando utilizado em ferramentas para trabalhos a quente.

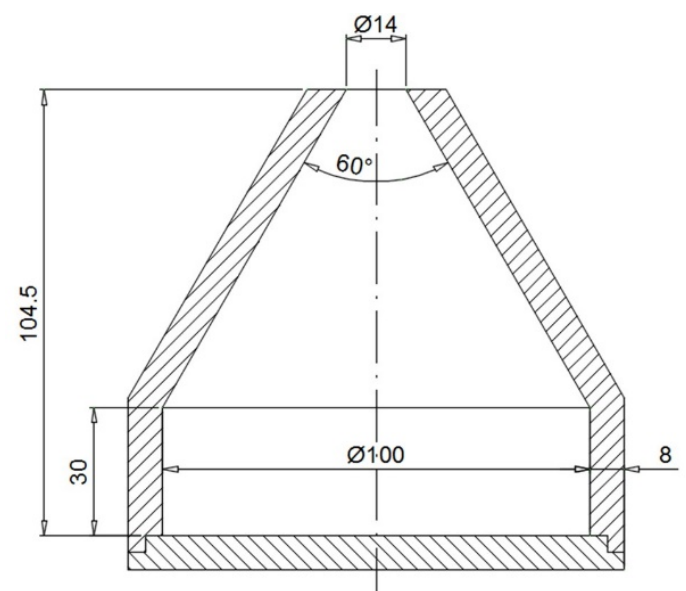

Figura 3. Desenho esquemático da coquilha Tatur.

Fonte: Ahdollahi (1998, p.48) - adaptado - unid: mm.

Aliga AISi7Cu3Mg foi utilizada para produção das amostras, com temperatura de $710 \pm 5{ }^{\circ} \mathrm{C}$ e densidade de $2,68 \mathrm{~g} / \mathrm{cm}^{3}$. A análise química da amostra é apresentada na Tabela 1. 
Tabela 1. Composição química da liga AlSi7Cu3Mg

\begin{tabular}{ccccccccc}
\hline $\begin{array}{c}\mathrm{Si} \\
\%\end{array}$ & $\begin{array}{c}\mathrm{Fe} \\
\%\end{array}$ & $\begin{array}{c}\mathrm{Cu} \\
\%\end{array}$ & $\begin{array}{c}\mathrm{Mn} \\
\%\end{array}$ & $\begin{array}{c}\mathrm{Mg} \\
\%\end{array}$ & $\begin{array}{c}\mathrm{Cr} \\
\mathbf{p p m}\end{array}$ & $\begin{array}{c}\mathrm{Zn} \\
\%\end{array}$ & $\begin{array}{c}\mathrm{Sr} \\
\mathbf{p p m}\end{array}$ & $\begin{array}{c}\mathrm{Pb} \\
\mathbf{p p m}\end{array}$ \\
\hline 7,16 & 0,543 & 3,4 & 0,435 & 0,352 & 152 & 0,431 & 25 & 375 \\
\hline
\end{tabular}

Fonte: Autor (2014).

\subsection{Métodos de dosagem}

Os experimentos foram desenvolvidos com a utilização de três métodos distintos de dosagem, o primeiro, denominado "método de dosagem direta", consiste no vazamento do metal da concha, diretamente para a cavidade da coquilha Tatur, como representado na Figura 4. A vazão e a dosagem do metal são dependentes da habilidade do operador.

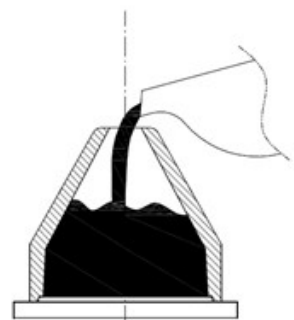

(a)

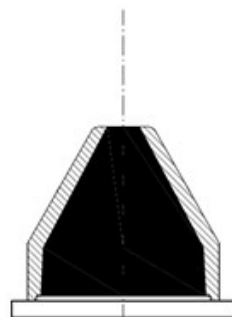

(b)

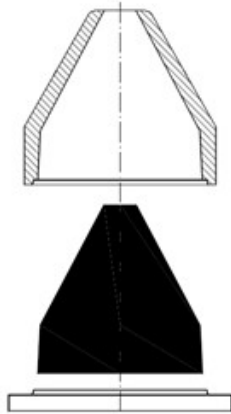

(c)

Figura 4. Vazamento em coquilha Tatur pelo método de dosagem direta.

Fonte: Autor (2015).

Os outros dois métodos foram efetuados com auxílio de dispositivo dosador, conforme Figura 5, onde o metal é vazado na bacia de vazamento (b) e posteriormente o obturador (c) é retirado para o escoamento do metal para a cavidade da coquilha (e).
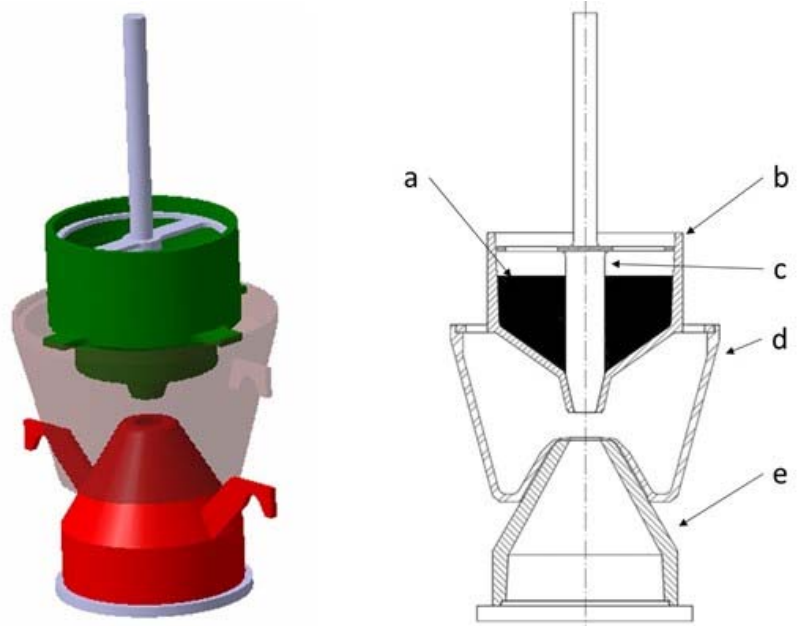

Figura 5. Esquema da coquilha Tatur com dispositivo de dosagem proposto:

(a) Alumínio líquido; (b) Bacia de vazamento; (c) Obturador do metal;

(d) Bacia de metal excedente e (e) Coquilha Tatur.

Fonte: Autor (2014). 
Os métodos de dosagem dinâmica e pré-dosagem se diferem na bacia de vazamento. Para o método de dosagem dinâmica, a capacidade volumétrica da bacia de vazamento é maior que o volume da cavidade da coquilha Tatur. Assim a dosagem do metal é definida pelo escoamento do metal excedente quando o molde se encontra cheio, conforme esquematizado na Figura 6 (c). Para o método de pré-dosagem, a bacia de vazamento foi modificada com a abertura de janelas laterais para a drenagem do metal excedente antes da retirada do obturador, conforme a Figura 7 (a). Assim, o volume de metal que fica contido na bacia de vazamento é ligeiramente menor que o volume da cavidade da coquilha Tatur.

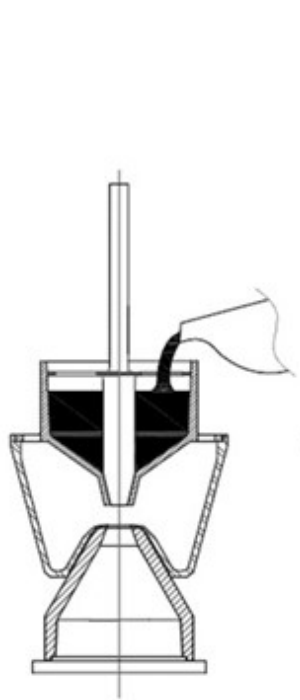

(a)

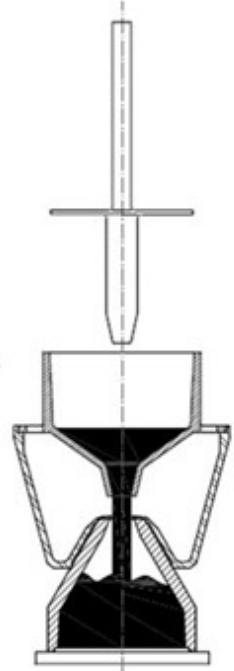

(b)

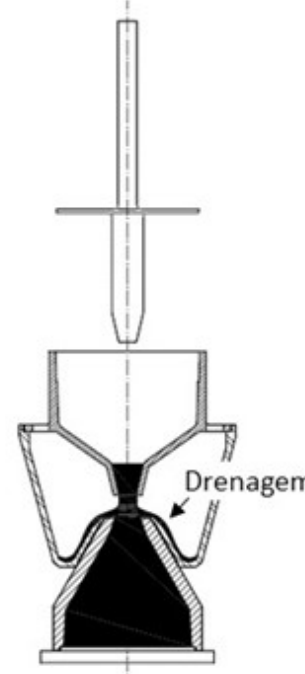

(c)

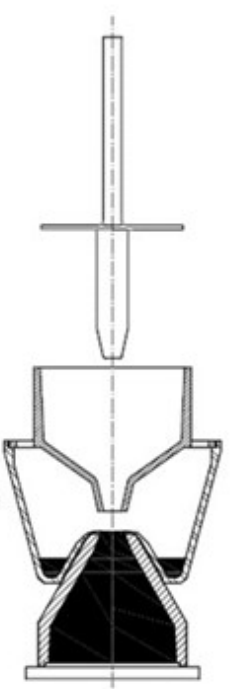

(d)

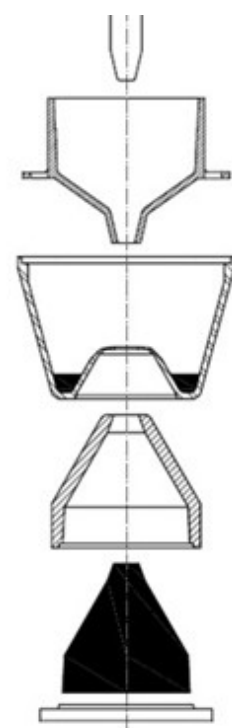

(e)

Figura 6. Método de dosagem dinâmica para a coquilha Tatur.

Fonte: Autor (2015).

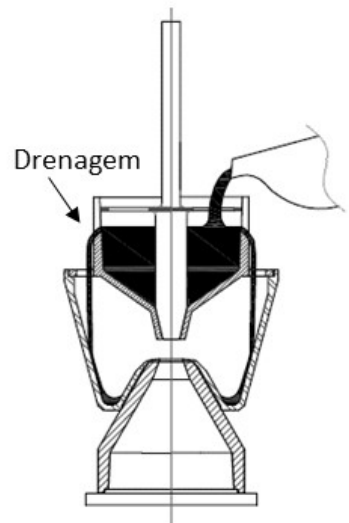

(a)

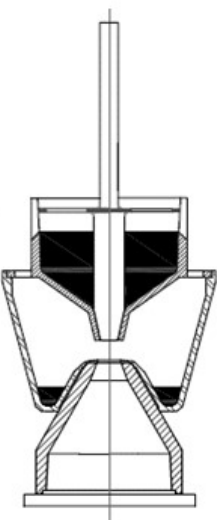

(b)

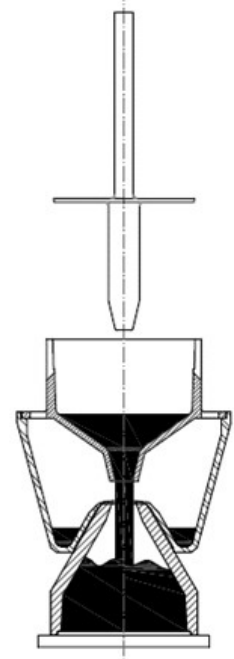

(c)

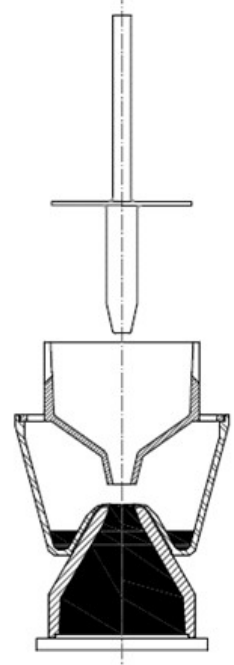

(d)

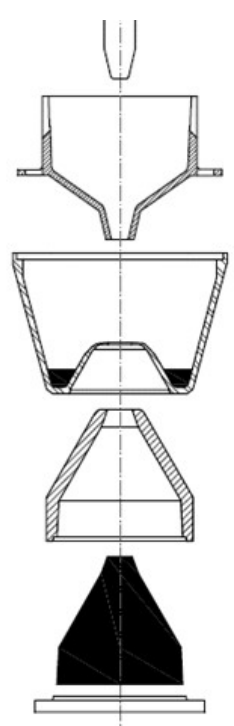

(e)

Figura 7. Método de Pré-dosagem para a coquilha Tatur.

Fonte: Autor (2015). 


\subsection{Avaliações dos métodos de dosagem}

As metodologias de dosagem direta, dosagem dinâmica e pré-dosagem foram comparadas, visando compreender os efeitos das vazões do metal líquido e suas dosagens sobre as morfologias das amostras, baseando-se no padrão morfológico teórico.

\subsubsection{Controle das vazões}

Sendo a vazão, a razão entre a massa de metal e o tempo de escoamento através do furo de alimentação até o completo enchimento da coquilha, a variável tempo é aquela que melhor indica o comportamento da vazão, pois o volume da cavidade do molde da coquilha é uma constante. Os experimentos foram filmados com câmera digital Sony DSC-W570, para posterior determinação dos períodos de tempo, controlados em software Windows Live Movie Maker. Os dados foram agrupados e tratados estatisticamente.

\subsubsection{Controle das dosagens}

As constatações das variações de dosagem foram obtidas por meio do controle das massas dos corpos de prova produzidos. Para a pesagem, uma balança SHIMADZU UX6200 foi utilizada. Os resultados foram agrupados e tratados estatisticamente conforme o método de dosagem utilizado.

\subsubsection{Avaliação das morfologias}

As morfologias das amostras foram analisadas, considerando a suas coerências com o padrão morfológico teórico. Essas observações foram também tratadas, levando em consideração o agrupamento por processos e correlações com as vazões e dosagens.

\section{RESULTADOS E DISCUSSÃO}

\subsection{Tempo de vazamento}

Os tempos de vazamento foram controlados e disposto conforme apresentado na Tabela 2. Para cada processo, as médias e desvios padrão dos tempos foram calculados para análise dos resultados e são definidas a seguir:

tDDin [s] = tempo médio dos vazamentos: dosagem dinâmica

tDDir [s] = tempo médio dos vazamentos: dosagem direta

tPréD [s] = tempo médio dos vazamentos: pré-dosagem

otDDin [s] = desvio padrão dos tempos de vazamento: dosagem dinâmica

otDDir [s] = desvio padrão dos tempos de vazamento: dosagem direta

otPréD [s] = desvio padrão dos tempos de vazamento: pré-dosagem

Os vazamentos efetuados com o dispositivo dosador pelos métodos de dosagem dinâmica e pré-dosagem apresentaram resultados similares, enquanto se diferenciaram consideravelmente dos tempos obtidos pelo método de dosagem direta. O dispositivo dosador proporcionou para ambos os métodos, a redução dos tempos médios de vazamento a menos de um terço, quando comparado ao processo efetuado pelo método de dosagem direta, com medias de tDDin $=4,00 \mathrm{~s}$, tPréD $=4,35 \mathrm{~s}$ e tDDir $=15,25$ s respectivamente. Houve também a redução da dispersão dos tempos de vazamento, indicados pelos desvios padrão de otDDin $=0,57$ s e otPréD $=0,46$ s para os métodos que utilizam o dispositivo dosador e otDDir =1,32 s, para dosagem direta. 
Tabela 2. Tempos de vazamento obtidos pelos métodos de dosagem dinâmica, pré-dosagem e

\section{Dosagem dinâmica}

\begin{tabular}{cc}
\hline$N^{\circ}$ & $\begin{array}{c}\text { Tempo de } \\
\text { vazamento (s) }\end{array}$ \\
\hline 1 & 3,40 \\
\hline 2 & 3,37 \\
\hline 3 & 4,45 \\
\hline 4 & 3,94 \\
\hline 5 & 4,95 \\
\hline 6 & 4,10 \\
\hline 7 & 3,80 \\
\hline \multicolumn{2}{c}{$\underline{t}_{\text {DDin }}=4,00$} \\
\hline \multicolumn{2}{c}{$\sigma_{\text {tDDin }}=0,57$} \\
\hline
\end{tabular}

dosagem direta

\begin{tabular}{cc}
\hline \multicolumn{2}{c}{ Pré-dosagem } \\
\hline $\mathrm{N}^{\circ}$ & $\begin{array}{c}\text { Tempo de } \\
\text { vazamento (s) }\end{array}$ \\
\hline 8 & 4,93 \\
\hline 9 & 3,78 \\
\hline 10 & 4,10 \\
\hline 11 & 3,82 \\
\hline 12 & 4,61 \\
\hline 13 & 4,40 \\
\hline 14 & 4,81 \\
\hline \multicolumn{2}{c}{ țréD $=4,35$} \\
\hline \multicolumn{2}{c}{$\sigma_{\text {tPréD }}=0,46$} \\
\hline
\end{tabular}

\begin{tabular}{cc}
\hline \multicolumn{2}{c}{ Dosagem direta } \\
\hline $\mathrm{N}^{\circ}$ & $\begin{array}{c}\text { Tempo de } \\
\text { vazamento (s) }\end{array}$ \\
\hline 15 & 16,63 \\
\hline 16 & 16,19 \\
\hline 17 & 14,15 \\
\hline 18 & 15,81 \\
\hline 19 & 15,25 \\
\hline 20 & 16,12 \\
\hline 21 & 12,93 \\
\hline \multicolumn{2}{c}{ t.dir $=15,25$} \\
\hline \multicolumn{2}{c}{$\sigma_{\text {tDDir }}=1,32$} \\
\hline \multicolumn{2}{c}{}
\end{tabular}

Fonte: Autor (2015).

Os métodos de dosagem dinâmica e pré-dosagem se mostraram semelhantes, quanto aos resultados dos tempos de vazamento e desvios padrão. Esses fatores são importantes para obtenção de amostras que reproduzam as morfologias dentro dos conceitos teóricos, como está concluído no tópico 3.3 "Morfologia das amostras".

\subsection{Dosagem}

Como exibido na Tabela 3, as massas das amostras foram controladas e organizadas conforme cada método de dosagem. As médias e desvios padrão das massas foram definidos como a seguir:

MDDin [g] = média das massas das amostras: dosagem dinâmica

MDDir $[\mathrm{g}]$ = média das massas das amostras: dosagem direta

MPréD $[\mathrm{g}]$ = média das massas das amostras: pré-dosagem

oMDDin [g] = desvio padrão das massas das amostras: dosagem dinâmica

oMDDir [g] = desvio padrão das massas das amostras: dosagem direta

oMPréD [g] = desvio padrão das massas das amostras: pré-dosagem

Variações entre os resultados de dosagem dos três métodos propostos foram encontradas. A menor dispersão ocorreu pelo método de dosagem dinâmica, com desvio padrão de oMDDin $=5,87$, enquanto a maior ocorreu pelo método de prédosagem, com desvio padrão de $\sigma \mathrm{MPré} D=21,18$. O método de dosagem direta apresentou dispersão $\sigma$ MDDir $=12,21$, situada entre os outros dois métodos.

Essas variações podem ser explicadas por observações feitas durante a produção das amostras. Para os processos de dosagem dinâmica e dosagem direta, as massas das amostras são determinadas durante o transbordamento do metal excedente no topo da coquilha. Quanto no processo de vazamento pelo método de pré-dosagem, o metal é dosado na bacia de vazamento e permanece ali durante o período necessário para que o operador possa depositar a concha de vazamento e voltar para a retirada do obturador. Esse período é suficiente para que se inicie um processo de solidificação na interface entre o metal e as paredes da bacia de vazamento. Assim, uma certa massa do metal dosado permanece em estado sólido na bacia de vazamento. A 
quantidade de metal remanescente varia conforme o tempo de permanência do metal e as temperaturas iniciais da liga e da bacia.

Tabela 3. Dosagens de metal obtidas pelos métodos de dosagem dinâmica, pré-dosagem e dosagem direta

\begin{tabular}{cc}
\hline \multicolumn{2}{c}{ Dosagem dinâmica } \\
\hline $\mathrm{N}^{\mathrm{o}}$ & Massa $(\mathrm{g})$ \\
\hline 1 & 1234 \\
\hline 2 & 1230 \\
\hline 3 & 1221 \\
\hline 4 & 1231 \\
\hline 5 & 1240 \\
\hline 6 & 1237 \\
\hline 7 & 1234 \\
\hline \multicolumn{3}{c}{ M } & DDin & $=1232$ \\
\hline \multicolumn{3}{c}{$\sigma_{\text {MDDin }}=5,87$} \\
\hline
\end{tabular}

\begin{tabular}{cc}
\hline \multicolumn{2}{c}{ Pré-dosagem } \\
\hline $\mathrm{N}^{\circ}$ & Massa $(\mathrm{g})$ \\
\hline 8 & 1198 \\
\hline 9 & 1201 \\
\hline 10 & 1203 \\
\hline 11 & 1164 \\
\hline 12 & 1217 \\
\hline 13 & 1233 \\
\hline 14 & 1210 \\
\hline \multicolumn{3}{c}{$\underline{M}_{\text {PréD }}=1204$} \\
\hline \multicolumn{2}{c}{$\sigma_{\text {MPréD }}=21,18$} \\
\hline
\end{tabular}

\begin{tabular}{|c|c|}
\hline \multicolumn{2}{|c|}{ Dosagem direta } \\
\hline $\mathrm{N}^{\circ}$ & Massa $(g)$ \\
\hline 15 & 1230 \\
\hline 16 & 1246 \\
\hline 17 & 1257 \\
\hline 18 & 1227 \\
\hline 19 & 1255 \\
\hline 20 & 1255 \\
\hline 21 & 1246 \\
\hline \multicolumn{2}{|c|}{$\underline{M}_{\mathrm{DDir}}=1245$} \\
\hline
\end{tabular}

Fonte: Autor (2015)

O método de pré-dosagem proporcionou amostras com maiores dispersões de massas, porém as variações encontradas não são suficientes para comprometer negativamente a morfologia dessas. Essa análise pode ser vista no tópico seguinte, 3.3 "Morfologia das amostras".

\subsection{Morfologia das amostras}

São exibidas na Figura 8 as secções de três amostras típicas dos lotes obtidos pelos métodos de dosagem dinâmica (a), pré-dosagem (b) e dosagem direta (c), respectivamente dispostas em perfil de uma máscara que reproduz a geometria da secção da cavidade interna do molde Tatur em escala 1:1.

Inicialmente, observa-se que as amostras (a) e (b), produzidas com dispositivo dosador, se assemelham por apresentarem rechupes em vale, enquanto para amostra (c), esse não se encontra. Os rechupes verticais das amostras (a) e (b) também seguiram a mesma tendência para os dois métodos que utilizaram dispositivo dosador, sendo mais acentuados e com as cristas mais bem definidas, quando comparados à amostra (c).

A contração geral das três amostras se assemelharam, a começar pelas geometrias cilíndricas situadas nas partes inferiores dessas, que mantiveram seus perfis com angulações próximas ao perfil representativo da coquilha, apresentando pouca redução dos seus diâmetros. A parte cônica das três amostras são similares quanto à diferença de angulação entre essas e o perfil teórico da coquilha, porém há uma grande diferença no perfil do topo da amostra (c), que se apresenta mais prolongado e deformado. Isso têm correlação com os mesmos fenômenos que levaram às anomalias na formação dos rechupes em vale e vertical. 


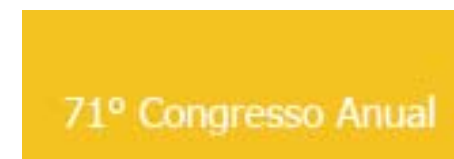

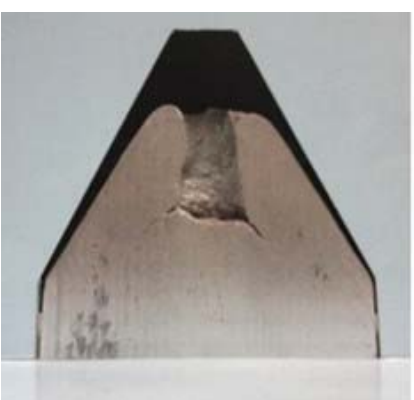

(a)

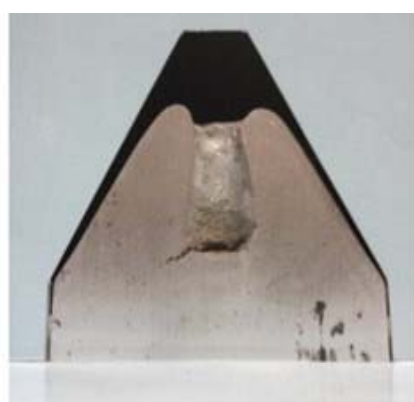

(b)

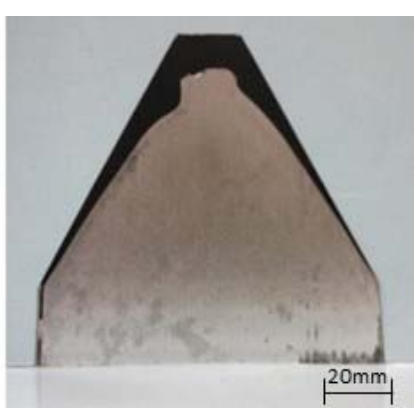

(c)

Figura 8. Amostras Tatur resultantes de três métodos distintos de dosagem:

(a) dosagem dinâmica, (b) pré-dosagem, (c) dosagem direta.

Fonte: Autor (2015).

A Tabela 4 exibe as avaliações qualitativas dos resultados da vazão, dosagem e morfologia, considerando as médias, desvios padrão e reprodução morfológica conforme o padrão teórico. Verifica-se que a vazão e a morfologia apresentam as mesmas classificações qualitativas e se diferem da dosagem direta. Assim, pode-se inferir que a vazão é mais influente na formação morfológica das amostras, que a dosagem.

Tabela 4. Relação qualitativa entre vazão, dosagem e morfologia das amostras Tatur pelos três métodos

\begin{tabular}{lccc} 
& Dosagem dinâmica & Pré-dosagem & Dosagem direta \\
\hline Vazão & + & + & - \\
\hline Dosagem & + & - & + - \\
\hline Morfologia & + & + & - \\
\hline
\end{tabular}

Fonte: Autor (2015)

\section{CONCLUSÃO}

O método Tatur com a utilização do dispositivo de dosagem proporcionou resultados relevantes e consistentes para a caracterização da capacidade de alimentação das ligas.

O dispositivo de dosagem proposto reduziu as dispersões do processo, garantindo maior repetitividade e proporcionando coerência morfológicas das amostras.

Os métodos propostos favoreceram a transferência do metal líquido da concha para a coquilha, pois a área de recebimento da liga no dispositivo é ampliada e o metal pode ser vazado com maior facilidade.

Foi verificado que a variável da vazão tem grande influência nas morfologias das amostras. Maiores vazões proporcionam melhores resultados.

A dosagem do metal pelo método de dosagem dinâmica proporcionou menor dispersão para o processo, que o método de pré-dosagem, apresentando sucessivamente médias e desvios padrão de: MDDin $=1232 \mathrm{~g}$ e $\sigma \mathrm{MDDin}=5,87 \mathrm{~g}$; MPréD $=1204 \mathrm{~g}$ e $\sigma \mathrm{MPréD}=21,18 \mathrm{~g}$. Essas diferenças não se mostraram impactantes na formação morfológica das amostras, pois seus resultados foram similares. 


\section{Agradecimentos}

Nemak do Brasil (Betim), FAPEMIG e FINEP pelo apoio financeiro ao projeto.

\section{REFERÊNCIAS}

1 Campbell J. Castings: the new metallurgy of cast metals. 2. ed. Birmingham: Elsevier, 2003. 337p.

2 Brůna M.,Sládek A. Hydrogen analysis and effect of filtration on final quality of castings from aluminum alloy AISi7Mg0,3. Foundry Commission of the Polish Academy of Sciences. Žilina, Slovakia, ISSN (1897-3310) v.11 Special Issue 1/2011, 2011.

3 La-Orchan W. Melt treatment effects on porosity and impact strength in hypoeutectic aluminum silicon alloy [dissertação de mestrado]. Montreal: Department of Mining and Metallurgical Engineering, McGill University, 1991.

4 Couper M. J.,Dahle A. K. Effect of $\mathrm{Sr}$ and $\mathrm{Na}$ on the castability of foundry alloy A356.2, In: Australian Foundry Institute National. Proceedings 35th Australian Foundry Institute National Conference. Out. 2004, Adelaide.

5 Ahdollahi A. Effect of $\mathrm{Ca}$ as a modifier in hypoeutectic Al-Si alloys [dissertação de mestrado]. Toronto: McGill University. 1998

6 AMERICAN SOCIETY FOR TESTING AND MATERIALS. ASTM B179: aluminum alloys in ingot and molten forms for castings from all casting process. West Conshohocken, USA, 2011. 